\title{
Outcome of laparoscopic surgeries during pregnancy for non-obstetric emergencies
}

\author{
Sunitha H. B. ${ }^{1}$, Vidya V. Bhat ${ }^{1}$, Ravishankar Bhat ${ }^{2}$, Nisha E. ${ }^{1}$, K. M. Guddy ${ }^{1}$ \\ ${ }^{1}$ Department of Obstetrics and Gynaecology, ${ }^{2}$ Department of Gastro Intestinal Surgery, Radhakrishna Multispecialty \\ Hospital/IVF Center, Bangalore, Karnataka, India
}

Received: 02 July 2016

Accepted: 05 August 2016

\author{
*Correspondence: \\ Dr. Sunitha H. B., \\ E-mail: suni.hb14obg@gmail.com
}

Copyright: ( $)$ the author(s), publisher and licensee Medip Academy. This is an open-access article distributed under the terms of the Creative Commons Attribution Non-Commercial License, which permits unrestricted non-commercial use, distribution, and reproduction in any medium, provided the original work is properly cited.

\begin{abstract}
Background: This study was conducted to evaluate the surgical and obstetric outcome, safety and feasibility of various laparoscopic surgeries for non-obstetric indications in pregnancy.

Methods: We did a retrospective analysis of 18 pregnant patients who underwent laparoscopic surgeries. Study period was from October 2013 till September 2015 conducted in Radhakrishna multispeciality hospital /IVF center Bangalore. Patients operated are 6 cases cholicystectomy, 6 cases appendicectomy, 5 adnexal mass removals, one salpingectomy for heterotopic pregnancy resulted from ART. All patients were between 11 to 32 weeks of gestation, with mean gestational age $21 \pm 6.5$ weeks at the time of surgery and mean duration of surgery was $46 \pm 16.3$ minutes.

Results: All eighteen pregnant patients had uneventful hospital courses after laparoscopic procedures. Mean duration of hospital stay after surgery was $43 \pm 8.5$ hours. One pregnancy was terminated at 11 weeks for suspected ovarian malignancy and 16 delivered full-term babies without complications, one patient delivered preterm at 35 weeks with NICU admission. The mean birth weight at the time of delivery was $2.8 \pm 550$ gms. There was no maternal morbidity or mortality, or any identifiable neonatal birth defect. No conversion to laparotomy required in any case.

Conclusions: Laparoscopic surgeries can be done in any trimester of pregnancy, but more safe and feasible during the second and early third trimester of pregnancy. Laparoscopic surgeries are as safe as laparotomy in the hands of experienced laparoscopic surgeon with no deleterious effects on either mother or fetus.
\end{abstract}

Keywords: Laparoscopic surgery in pregnancy, Non-obstetric emergency surgeries during pregnancy, Surgeries during pregnancy

\section{INTRODUCTION}

Pregnancy association with non-obstetric medical problems is not uncommon. Approximately 1 in 500 to 1 in 700 women will require non-obstetrical surgery during their pregnancies. ${ }^{1}$ Incidence of non-obstetrical surgeries during pregnancy is $0.75 \%-2 \% .^{2}$ Acute abdominal conditions in pregnancy are common as in non-pregnant women, with acute appendicitis commonest followed by cholelithiasis, cholicystitis, adnexal masses most commonly ovarian cysts, intestinal obstruction abdominal trauma are other rare causes. ${ }^{4}$ Though heterotopic pregnancy was rare in older days, but now due to ART incidence is increasing. With recent advancements in laparoscopic surgical techniques, its use in pregnancy is becoming widely safe and acceptable. But thorough knowledge of the anatomical aspects, physiological changes in pregnancy, risks and benefits, complications of laparoscopic surgery and its management is mandatory.

We have managed in our centre eighteen cases of pregnant patients with symptomatic co morbidities laparoscopically with favourable surgical and obstetric outcome. The patient's operated were six cases laparoscopic cholicystectomy, six cases of 
appendicectomy, five Adnexal mass removals and one salpingectomy for heterotopic pregnancy. Heterotopic pregnancy resulted from IVF-ET procedure.

\section{METHODS}

Our study was retrospective analysis conducted from October 2013 September 2015 in Radhakrishna multispecialty hospital Bangalore.

\section{Inclusion criteria}

- Acute cholicystitis

- Acute appendicitis

- Asymptomatic Ovarian cysts $>7 \mathrm{~cm}$

- Ovarian cysts with torsion, acute pain abdomen.

\section{Exclusion criteria}

- Asymptomatic simple ovarian cysts $<6-7 \mathrm{~cm}$

- Asymptomatic cholilithiais

- Acute appendicular mass

- Red degeneration of fibroids

- Surgeries combined with LSCS.

Informed consent was taken from all patients. Ethical approval was obtained from the local institutional review board. Patients operated were in the gestational period ranging from 11 weeks to 32 weeks with no obstetrical complications. Preoperative routine haematological and serological tests were done. Intraoperative positioning of patients during cholicystectomy surgery was supine position with left lateral tilt of 15 degrees to prevent aortocaval compression. Position during pelvic procedures was 20 degrees trendlenberg position. General anaesthesia was used in all cases. Meticulous preoxygenation was done to prevent hypoxia. ${ }^{2}$ Antacid prophylaxis and rapid sequence induction done with cricoid pressure, cuffed endotracheal tube used to reduce risk of aspiration. Antibiotic prophylaxis was given intraoperatively at the time of induction of anesthesia. ${ }^{2}$ $\mathrm{PaCO} 2$ was closely monitored by the routine use of end tidal carbon dioxide (et co2) monitoring using capnography. $^{2}$ Various laparoscopic entry techniques such as supraumbilical, palmer's point, or left upper quadrant (LUQ) entry was used in pregnancy depending on size of uterus, previous abdominal surgeries, surgeon's expertise. Veress needle or open Hassan technique was used and pneumoperitoneum created by insufflation of carbon dioxide until an abdominal pressure of 12-14 mm $\mathrm{Hg}$ was achieved. ${ }^{1}$ The lowest pressure pneumoperitoneum $(10-14 \mathrm{mmHg})$ was used in all cases. ${ }^{1}$ Primary $11 \mathrm{~mm}$ telescopic port introduced blindly and the peritoneal cavity was visualized with a 10 mm 30 degree telescope, and secondary ports were inserted under direct vision at different quadrants of abdomen depending upon height of uterus and surgery performed.
Surgical procedures were performed according to standard laparoscopic techniques. At the end of the surgery ovarian tissue, gall bladder and salpingectomy specimen retrieved through the endobag and appendix retrieved through $10 \mathrm{~mm}$ telescopic port. Ports closed. The average duration of surgery was $46 \pm 16.3$ minutes.

FHR monitoring was done pre and postoperatively in second and third trimester patients. ${ }^{1}$ No prophylactic tocolysis was used in any of the case. ${ }^{1} 2$ patients received antenatal steroid prophylaxis with gestational age of 28 weeks and more. Intraoperative and postoperative pneumatic compression devices and early postoperative ambulation was given for prophylaxis of deep venous thrombosis. ${ }^{1}$ Post-operatively patients were discharged after 36-48 hours after surgery. Mean duration of hospital stay was $43 \pm 8.5$ hours. Patients were regularly followed up in obstetric opd in antenatal period to detect at the earliest any untoward complications. Mode of delivery, obstetric and neonatal outcome analyzed. Postnatal follow up done till one year of age in pediatrics opd.

\section{RESULTS}

During the 24 months study period, 18 pregnant women underwent surgery for various non-obstetric conditions. Mean gestational age at the time of surgery was $21 \pm 6.5$ weeks. Mean duration of surgery was $46 \pm 16.3$ minute. No intraoperative and postoperative maternal or foetal complications were seen. Mean duration of hospital stay after surgery was $43 \pm 8.5$ hours. One pregnancy was terminated at 11 weeks in multiparous women on request in which the histopathological examination showed borderline ovarian tumor of serous variety. 3 ovarian mass were simple ovarian cysts, in which one patient had presented with acute abdomen due to torsion of ovarian cyst and one benign cystic teratoma was reported. One patient with h/o primary infertility 10 years was treated by ART (IVF-ET) was diagnosed to have heterotopic pregnancy at 7 weeks pregnancy by TVS. Patient was asymptomatic and condition was diagnosed accidentally in scan. Scan revealed a right tubal unruptured ampullary ectopic pregnancy with healthy on-going intrauterine pregnancy. Patient underwent laparoscopic right salpingectomy with continuation of intrauterine pregnancy.

Table 1: General information of patients.

\begin{tabular}{|ll|}
\hline Age (range) & 21- 36 years \\
\hline BMI & 20-31 \\
\hline $\begin{array}{l}\text { Gestational age } \\
\text { (trimester) }\end{array}$ & I TM-3 \\
& II TM-11 \\
III TM-4 \\
\hline Previous surgeries & $\begin{array}{l}\text { Previous caesarian section- } 4 \\
\text { DHL-1, No surgeries -13 }\end{array}$ \\
\hline
\end{tabular}

BMI-body mass index; DHL-diagnostic hysterolaparoscopy. 
Table 2: Laparoscopic surgeries.

\begin{tabular}{|ll|}
\hline Laparoscopic Surgeries & No. \\
\hline Cholicystectomy & 6 \\
\hline Appendicectomy & 6 \\
\hline Adnexal mass removal & 5 \\
\hline Salpingectomy & 1 \\
\hline
\end{tabular}

Table 3: Obstetric outcome.

\begin{tabular}{|ll|}
\hline Mode of delivery & $\begin{array}{l}\text { Termination of } \\
\text { pregnancy - 1, PTND - 1, } \\
\text { FTND - 10, LSCS - 6 }\end{array}$ \\
\hline Birth weight & $2.8 \pm 550$ gms. \\
\hline $\begin{array}{l}\text { Complications } \\
\text { (intrapartum/postpartum) }\end{array}$ & Nil \\
\hline
\end{tabular}

Table 4: Surgical outcome.

\begin{tabular}{|ll|}
\hline Adnexal masses & No. \\
\hline Simple ovarian cyst & 3 \\
\hline Benign cystic teratoma & 1 \\
\hline Borderline s ovarian tumor & 1 \\
\hline Gall bladder/appendix & Inflammatory lesions \\
\hline
\end{tabular}

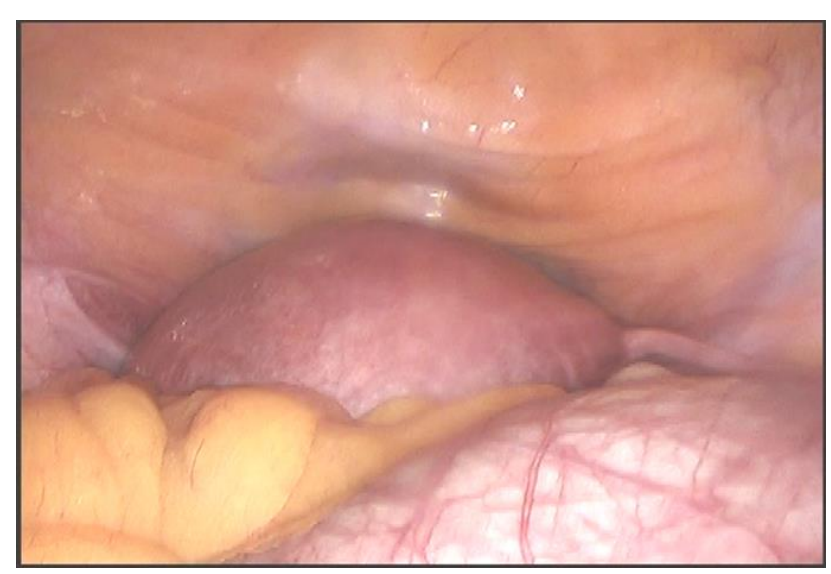

Figure 1: Large right simple ovarian cyst with 11 weeks pregnancy.

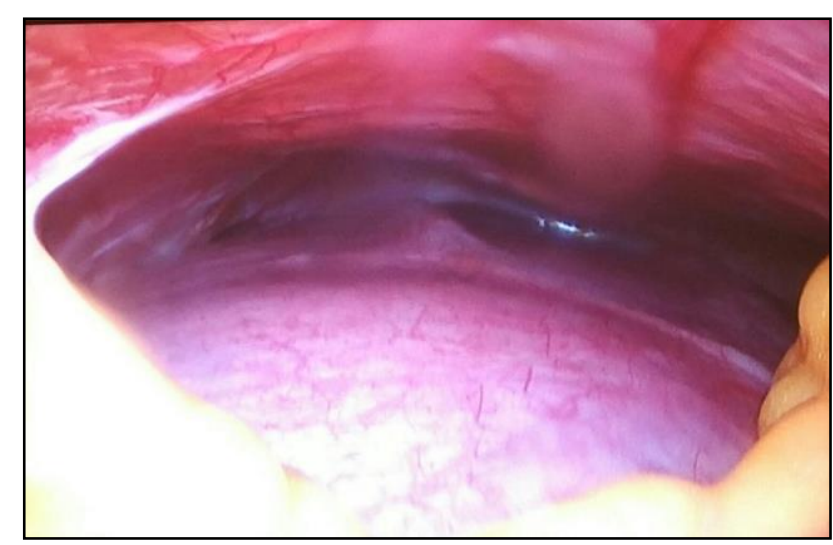

Figure 2: 26 week's gravid uterus with calculus cholicystitis in a case of previous caesarian section.

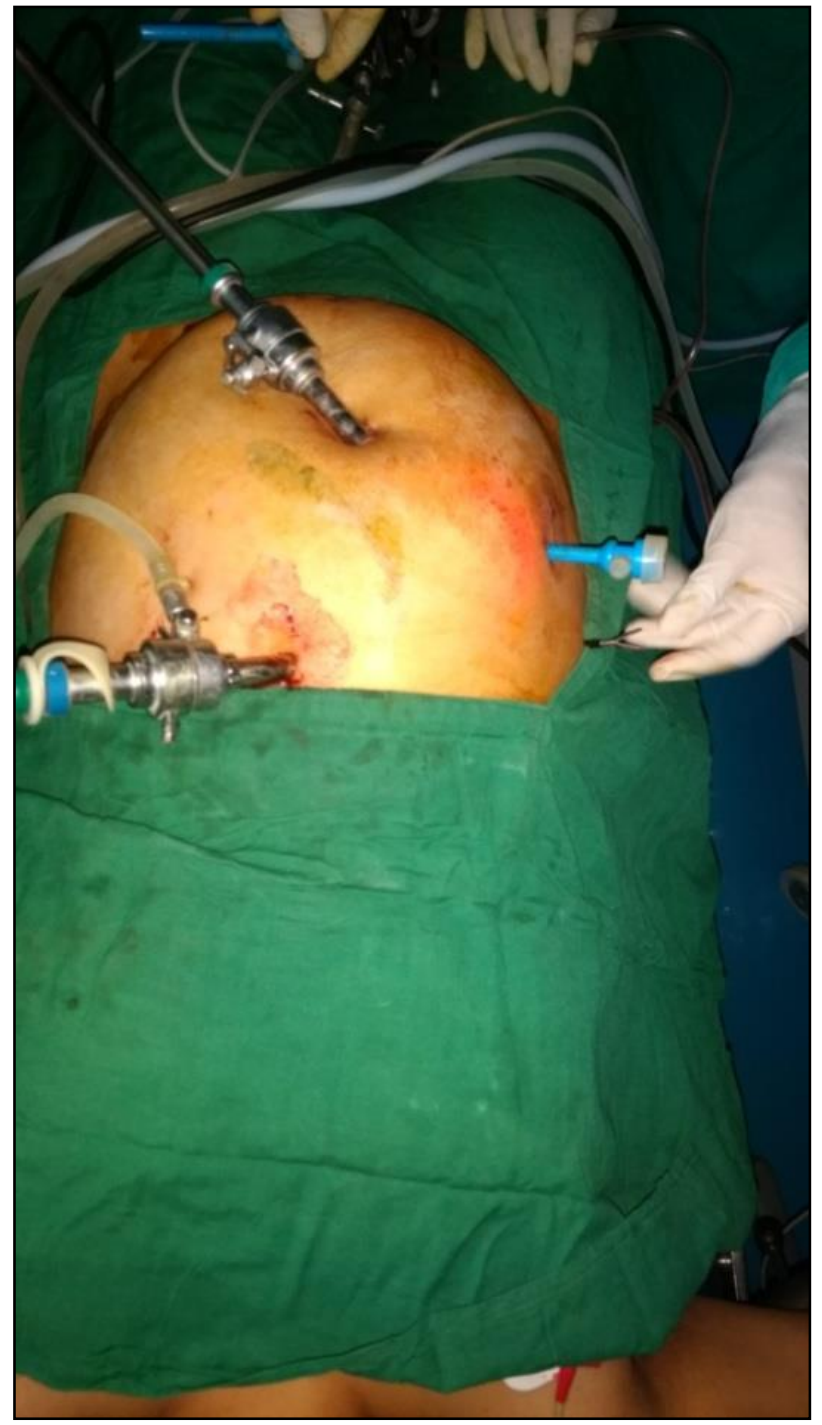

Figure 3: Port placement in case of cholicystectomy with 26 weeks gravid uterus.

No maternal morbidity and mortality was found. 16 patients had uneventful term deliveries and one patient had preterm delivery at 35 weeks gestation. 10 patients delivered vaginally and 6 patients had uneventful lower segment caesarian section for obstetric indications. One NICU admission due to preterm delivery at 35 weeks weighed $2.2 \mathrm{~kg}$ discharged after 72 hours. Mean birth weight at the time of birth was $2.8 \pm 550$ grams. Mothers and children were followed up for 12 months. No abnormalities found in milestones development.

\section{DISCUSSION}

In a large series, Lachman et al analyzed 518 pregnant patients undergoing surgery and found that laparoscopic cholecystectomy was the commonest (45\%), followed by adnexal surgery (34\%) and appendicectomy $(15 \%){ }^{4}$

Few SAGES guidelines for use of laparoscopy during pregnancy are highlighted below. 
- Initial access can be safely accomplished with an open or Hassan technique, verres needle or optical trocar. Intra-abdominal pressure should be sufficient to allow for adequate visualization

- Laparoscopy can be safely performed during any trimester of pregnancy

- Intra-operative end tidal $\mathrm{CO} 2$ monitoring by capnography should be used for monitoring

- Tocolytics should not be used prophylactically, but should be considered peri-operatively when signs of preterm labor are present in coordination with obstetric consultation.

Incidence of Adnexal mass complicating pregnancy is around $2 \%$. $^{1}$ Functional ovarian cysts, corpus luteal cysts and benign cystic teratomas, benign cystic adenoma contribute to two-third of cases. ${ }^{3,6}$ Torsion of the cyst complicates in $10-15 \%$ of adnexal masses. ${ }^{1}$ In 2 to $4 \%$ of cases chances of malignancy exists. ${ }^{3}$

SAGES guidelines 19 and 20: laparoscopy is safe and effective treatment in gravid patients with symptomatic ovarian cystic masses. ${ }^{1}$ It is recommended for both diagnosis and treatment of adnexal torsion unless clinical severity warrants laparotomy.

Appendicitis is encountered in $0.05 \%$ to $0.13 \%$ especially during second and third trimester of pregnancy. Diagnosis is difficult due to displacement of appendix by the gravid uterus. The incidence of fetal loss is $1.5 \%$ in uncomplicated cases and $35 \%$ in complicated ruptured appendix cases. ${ }^{5}$

SAGES guideline 17: laparoscopic appendectomy may be performed safely in any patients with suspicion of appendicitis (level II, grade B). ${ }^{1}$ It usually occurs in the second or third trimesters. Appendicitis occurs at the same rate in pregnant and non-pregnant women, but pregnant women have a higher rate of perforation.

Cholecystitis occurs in 1:1500-10,000 pregnancies. $^{7}$ Pregnancy is associated with increased gall bladder conditions due to changes in hormone levels especially progesterone. ${ }^{7}$ Increased cholesterol secretion, increased bile acid pool and cholistasis, decreased enterohepatic circulation are factors responsible for cholilithiasis and cholicystitis. $^{7,8}$

SAGES Guideline 15: laparoscopic cholicystectomy is the treatment of choice in the pregnant patient with gallbladder disease regardless of trimester (level II, grade B). ${ }^{1}$ If cholangiography is necessary, a lead shield should be placed over the entire infraumbilical area to protect the fetus.

Heterotopic pregnancy is a condition in which coexistence of intra and extra- uterine pregnancies occurs. It is a rare condition. With the widespread use of assisted reproductive techniques (ART), especially ovulation induction, the incidence may be as high as
$0.75-1.3 \%$ of obtained pregnancies. ${ }^{11}$ Careful serial transvaginal ultrasound should be done to rule out any ectopic pregnancies along with intrauterine pregnancy especially after intrauterine insemination and ART procedures to diagnose at the earliest to avoid devasting catastrophes such as rupture. ${ }^{9,10}$

\section{Risks associated with laparoscopic surgery are}

- Inadvertent injury and perforation of uterus during verres needle/optical trocar insertion

- Reduction in uterine blood flow due increase in intraabdominal pressure

- Preterm labour

- Hypercarbia to both mother and fetus ${ }^{11}$

- Miscarriages, Fetal bradycardia, fetal loss.

\section{Drawbacks of laparoscopic surgeries during pregnancy}

Technical difficulty of doing laparoscopy due to limitations of space and poor visualisation of operative field by the gravid uterus. Increased vascularity and hemodynamic alterations in pregnancy are other factors to keep in mind.

Best timing for surgeries during pregnancy is second trimester as there is less chance of miscarriages, low risk of teratogenicity, operative field is better, less risk of preterm labour. $^{12}$

\section{CONCLUSION}

Laparoscopic surgery can be safely performed in any trimester of pregnancy.

A fully equipped set up is necessary. Skilled and experienced laparoscopic surgeon, well trained staff is a must. Thromoprophylaxis has to be considered. Tocolytics and intra-partum foetal monitoring are required when necessary.

\section{Funding: No funding sources \\ Conflict of interest: None declared}

Ethical approval: The study was approved by the Institutional Ethics Committee

\section{REFERENCES}

1. Guidelines for diagnosis, treatment, and use of laparoscopy for surgical problems during pregnancy. Society of American Gastrointestinal and Endoscopic Surgeons, 2011. Available at http://www.sages.org/publications/guidelines/guideli nes-for-diagnosis-treatment-and-use-of-laparoscopyfor-surgical-problems-during-pregnancy/.

2. Hool A. Central Manchester Foundation Trust Anaesthesia in pregnancy for non-obstetric surgery anaesthesia tutorial of the week 185, 28th June 2010. Available 
http://www.frca.co.uk/Documents/185\%20Anaesthes ia\%20in\%20pregnancy\%20for\%20nonobstetric\%20surgery.pdf.

3. Ngu SF, Vincent YT, Pun TC. Surgical management of adnexal masses in pregnancy. JSLS. 2014;18:715 .

4. Lachman E, Schienfeld A, Voss E. Pregnancy and laparoscopic surgery. J Am Assoc Gynecol Laparosc. 1999;6:347-51.

5. Palanivelu C, Rangarajan M, MS. Laparoscopic appendectomy in pregnancy: a case series of seven patients. JSLS. 2006;10:321-5.

6. Sayer RA, Hoffman MS. A guide to management Adnexal masses in pregnancy. OBG Manag. 2007;19(3):27-44.

7. Amoli HA, Tavakoli H, Notash AY, Far MS. Laparoscopic cholicystectomy during pregnancy: a case series. J Min Access surg. 2008;1:9-14.
8. Chamogeorgakis T, Menzo EL, Smink RD, Feuerstein B, Fantazzio M, Kaufman J, et al. Laparoscopic cholicystectomy during pregnancy: three case reports. JSLS. 1999;3:67-9.

9. Duggal BS, Tarneja P, Sharma RK, Rath SK, Wadhwa VSM. Laparoscopic management of ectopic pregnancies. MJAFI. 2004;60:220-3.

10. Louis-Sylvestre C, Morice P, Chapron C, Dubuisson JB. Case report the role of laparoscopy in the diagnosis and management of heterotopic pregnancies. Human Reproduction. 1997;12(5):1100-2.

11. Goodman S. Perinatol S. Anesthesia for nonobstetric surgery in the pregnant patient. 2002;26(2):136-45.

12. Emergency General Surgery. The Pregnant Patient and Non-Obstetrical Emergency Surgery. Revised: February 6, 2004. Available at http://traumaburn.com/Protocols/PregnancyNonObst et.pdf.

Cite this article as: Sunitha HB, Bhat VV, Bhat R, Nisha E, Guddy KM. Outcome of laparoscopic surgeries during pregnancy for non-obstetric emergencies. Int J Reprod Contracept Obstet Gynecol 2016;5:3022-6. 